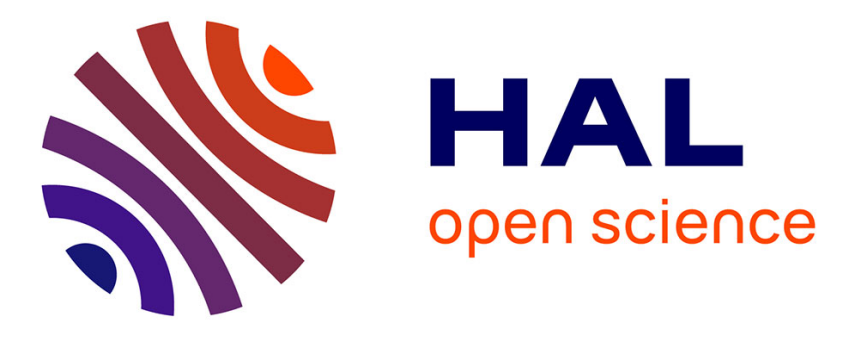

\title{
Physical analysis of the through-ligand long-distance magnetic coupling: spin-polarization versus Anderson mechanism
}

Thibaut Térencio, Roland Bastardis, Nicolas Suaud, Daniel Maynau, Jacques Bonvoisin, Jean-Paul Malrieu, C. J. Calzado, Nathalie Guihéry

\section{To cite this version:}

Thibaut Térencio, Roland Bastardis, Nicolas Suaud, Daniel Maynau, Jacques Bonvoisin, et al.. Physical analysis of the through-ligand long-distance magnetic coupling: spin-polarization versus Anderson mechanism. Physical Chemistry Chemical Physics, 2011, 13 (26), pp.12314-12320. 10.1039/c1cp20179d. hal-00864023

\section{HAL Id: hal-00864023 https://hal.science/hal-00864023}

Submitted on 19 Mar 2018

HAL is a multi-disciplinary open access archive for the deposit and dissemination of scientific research documents, whether they are published or not. The documents may come from teaching and research institutions in France or abroad, or from public or private research centers.
L'archive ouverte pluridisciplinaire HAL, est destinée au dépôt et à la diffusion de documents scientifiques de niveau recherche, publiés ou non, émanant des établissements d'enseignement et de recherche français ou étrangers, des laboratoires publics ou privés. 


\title{
Physical analysis of the through-ligand long-distance magnetic coupling: spin-polarization versus Anderson mechanism
}

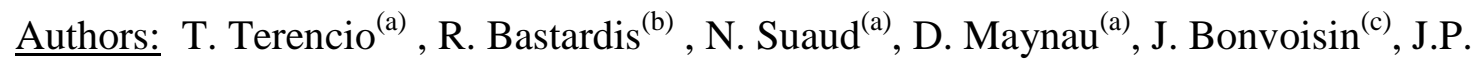
Malrieu $^{(\text {a) }}$, C. J. Calzado ${ }^{(\mathrm{d})}$ and N. Guihéry ${ }^{(\mathrm{a})}$

\section{Affiliations:}

(a) Laboratoire de Chimie et Physique Quantiques, Université de Toulouse III, 118, route de Narbonne, 31062 Toulouse, France

(b) Laboratoire de Mathématiques, Physique et Systèmes, Université de Perpignan, Via Domitia, 52 avenue Paul Alduy, 66860 Perpignan, France

(c) CEMES/CNRS, NanoSciences Group, BP 94347, 29 rue Jeanne Marvig, 31055 Toulouse cedex 4, France

(d) Departamento de Química Física, Universidad de Sevilla, Profesor García González s/n, 41012 Sevilla, Spain

\begin{abstract}
The physical factors governing the magnetic coupling between two magnetic sites are analyzed and quantified as functions of the length of the bridging conjugated ligand. Using wave-function-theory based $a b$ initio calculations, it has been possible to separate and calculate the various contributions to the magnetic coupling, i.e. the direct exchange, the spin polarization and the kinetic exchange. It is shown on model systems that while the Anderson mechanism brings the leading contribution for short-length ligands, the spin polarization dominates the through-long-ligand couplings. Since the spin polarization decreases more slowly than the kinetic exchange, highly spin polarizable bridging ligand would generate a good magneto-communication between interacting magnetic units.
\end{abstract}




\section{Introduction}

The electronic communication between two magnetic sites connected through bridges has been a topic of large interest in molecular electronics[1-4] and spintronics[5,6]. Much effort from experimentalists has been invested in the elucidation of the role of extended bridges between two interacting units, both concerning the electron transfer in mixed valence compounds (electro-communication) and the magnetic coupling of unpaired electrons in pure magnetic compounds (magneto-communication).

From a theoretical point of view, several works reviewed in references $[7,8]$ have been devoted to the methodology required to accurately reproduce the magnitude and sign of magnetic couplings in bi-nuclear compounds involving short bridges. Beyond the contribution to quantum chemistry computational techniques, these papers [9-11] illustrate how the required methodology is intimately related to the physical factors governing these effective interactions. In the line of these previous works, the electronic mechanisms involved in the charge or spin communication through extended bridging ligands have to be understood.

The decrease of $\mathrm{J}$ as a function of the conjugated-bridge length has been theoretically evidenced, using UDFT calculations in nytronil nitroxide diradicals[12] and dinitrenes[13]. In recent experimental and theoretical studies[14,15] performed on dinuclear copper(II) complexes connected through oligoacenebis(oxamate) or oligo-paraphenylenediamine bridging ligands, Unrestricted Density Fonctional Theory (UDFT) calculations support the occurrence of a spin polarization mechanism for the exchange interaction between the two distant unpaired electrons. This alternative channel for the propagation of the exchange coupling through extended ligand could exhibit a different decay with the inter-metallic distance than the kinetic exchange, and this could explain the previously commented discrepancy. The prevalence of the spin polarization mechanism was proposed on the basis of the appearance of non-negligible spin density on the bridge in the UDFT $M_{S}=0$ solution. This feature can only be considered as an indication since there is no spin density in the real singlet state and it does not give any information on the relative contributions of spin polarization and kinetic exchange to the resulting magnetic coupling.

Actually this work was also initially motivated by the unexpected behaviour found in a set of new phenylcyanamido-bridge dinuclear $\mathrm{Ru}$ complexes, recently synthesized and characterized[16] by one of us (JB). The mixed valent $\mathrm{Ru}(\mathrm{II}) \mathrm{Ru}(\mathrm{III})$ and homovalent paramagnetic $\mathrm{Ru}(\mathrm{III}) \mathrm{Ru}(\mathrm{III})$ forms of all the complexes were studied by ultra-violet-visible- 
near-infrared (UV-vis-NIR) and electron paramagnetic resonance (EPR) spectroscopy. Electronic communication was quantified by the hopping integral $\mathrm{V}_{\mathrm{AB}}$ (between metals $\mathrm{A}$ and B) extracted from intervalence transition measurements in the near IR area, and magnetic communication was quantified in terms of the exchange coupling interaction $\mathrm{J}$, accessible from the intensity of the EPR signal when varying temperature. $V_{A B}$ and $J$ versus intermetallic distances exhibit an exponential decay with two different laws. The slopes $\gamma$ and $\gamma^{\prime}$ of the $\ln \left(\left|\mathrm{V}_{\mathrm{AB}}\right|\right)$ and $\ln (\mathrm{J})$ lines respectively are such that $\gamma^{\prime} \approx \gamma / 2$. At the first glance, this relationship between the two parameters is quite surprising. Indeed, the superexchange magnetic interaction is usually described as resulting from the Anderson mechanism (see Section II) in which $\mathrm{J}$ scales as $\mathrm{t}^{2} / \mathrm{U}$ (kinetic exchange) where $\mathrm{t}$ and $\mathrm{U}$ are characteristics of the magnetic compound; $t$ is the inter-site hopping integral and $U$ the relative energy of the ionic form $\mathrm{A}^{-} \mathrm{B}^{+}$ resulting from a charge transfer between the metals. Neglecting the dependence of $U$ to the inter-metallic distance and making the assumption that the hopping integrals $t$ (in the homovalent magnetic system) and $\mathrm{V}_{\mathrm{AB}}$ (in the mixed-valent system) have the same exponential decay would lead to the relation $\gamma^{\prime}=2 \gamma$. While the elucidation of this unexpected behavior which would require the study of mixed valent systems is out of the scope of the present study, we will analyze the relative importance and decay laws of both the kinetic exchange and spin polarization contributions to the magnetic coupling.

This work takes benefit of the possibility offered by wave function based calculations to distinguish the effect of the configurations involved in each mechanism in order to evaluate their separate contributions and to analyze their decay with the inter-magnetic-site distance. Furthermore, the separation of both mechanisms enables us to quantify all effective interactions involved in the magnetic coupling, i.e. direct exchange, spin polarization, hopping integral $t$ and on-site electron repulsion $\mathrm{U}$.

In section II, the various physical factors governing the magnetic interaction are briefly summarized and the methodology required to accurately reproduce them is recalled. Section III is devoted to an ab initio study of the different contributions to the magnetic coupling as functions of the distance in model compounds. Conclusion and perspectives are presented in section IV. 


\section{Theory and computation}

The magnetic coupling is the leading effective interaction of spin Hamiltonians. For a binuclear system of spin $S=1 / 2$, the model space of this Hamiltonian is constituted of the two neutral valence bond determinants in which each magnetic sites has one unpaired electron. As shown in several papers, the microscopic origins of the magnetic coupling are multiple. This section recalls briefly the main physical factors that govern the magnitude and sign of this interaction $[9-11,18]$.

\section{II.a Physical factors governing the magnetic coupling: recalls}

For a centro-symmetric binuclear magnetic system, the local magnetic molecular orbitals (MOs) $\mathbf{a}$ on site A and $\mathbf{b}$ on site B are obtained from the gerade $\mathbf{g}$ and ungerade $\mathbf{u}$ MOs:

$$
\begin{aligned}
& a=\frac{g+u}{\sqrt{2}} \\
& b=\frac{g-u}{\sqrt{2}}
\end{aligned}
$$

It is important to recall that the quantum chemical optimization of the $\mathbf{g}$ and $\mathbf{u}$ orbitals results in delocalizations between the magnetic site and the ligand, i.e. the magnetic orbitals have tails on the ligand and reciprocally the ligand orbitals have tails on the magnetic sites. This feature has important consequences on the magnitude of the various contributions to the coupling described here-after.

Two main factors govern the magnetic coupling between these unpaired electrons:

i) The direct exchange $\mathrm{K}$ defined as:

$$
\mathrm{K}=\left\langle\mathrm{ab}\left|\mathrm{r}_{12}^{-1}\right| \mathrm{ba}\right\rangle
$$

This integral is always positive and hence favors a ferromagnetic coupling. It is large when the two a and b MOs present an important "differential overlap", i.e. when, despite their orthogonality, they both have significant amplitudes in some regions of space. This integral is expected to decrease rapidly with the distance between the two magnetic sites. Nevertheless the delocalization of the magnetic orbitals on the bridging ligand may lead to non-negligible values of this contribution. 
ii) The kinetic exchange. This contribution is brought by the delocalization of the electrons between the two magnetic MOs, i.e. the electron on site A may jump from a to $\mathbf{b}$ provided that the hopping integral $t$ between $\mathbf{a}$ and $\mathbf{b}$ is non-zero. This integral represents the interaction between the neutral valence-bond (VB) forms $|a \bar{b}|$ and $|b \bar{a}|(A \cdot B)$ and the ionic VB forms $|\mathrm{a} \overline{\mathrm{a}}|\left(\mathrm{A}^{-} \mathrm{B}^{+}\right)$and $|\mathrm{b} \overline{\mathrm{b}}|\left(\mathrm{A}^{+} \mathrm{B}^{-}\right)$. It is non-zero except for symmetry reasons. The on-site repulsion $\mathrm{U}$ is the energy difference between the neutral ungerade triplet and the ionic gerade singlet function. In the basis of the following neutral and ionic functions:

$$
\begin{aligned}
& \left|\phi_{N}\right|=\frac{1}{\sqrt{2}}(|a \bar{b}|+|b \bar{a}|) \\
& \left|\phi_{I}\right|=\frac{1}{\sqrt{2}}(|a \bar{a}|+|b \bar{b}|)
\end{aligned}
$$

the Hamiltonian matrix obtained for the two-magnetic-electron case is:

$$
\left(\begin{array}{cc}
2 \mathrm{~K} & 2 \mathrm{t} \\
2 \mathrm{t} & \mathrm{U}
\end{array}\right)
$$

where the triplet state energy is taken as zero of energy. The corresponding electronic mechanism, known as the Anderson mechanism [19] (see scheme 1), introduces ionic VB components in the wave functions of the lowest singlet state:

$$
{ }^{1} \Psi_{g}=\lambda \mid \text { core.g } \bar{g}|-\mu| \text { core. } u \bar{u}\left|=C_{N}\right| \text { core. }(a \bar{b}+b \bar{a})\left|+C_{I}\right| \text { core. }(a \bar{a}+b \bar{b}) \mid
$$

where core describes the product of the closed shell MOs and $\mathrm{C}_{\mathrm{N}}$ and $\mathrm{C}_{\mathrm{I}}$ are normalized coefficients, with $\left|\mathrm{C}_{\mathrm{N}}\right|>\left|\mathrm{C}_{\mathrm{I}}\right|$. 


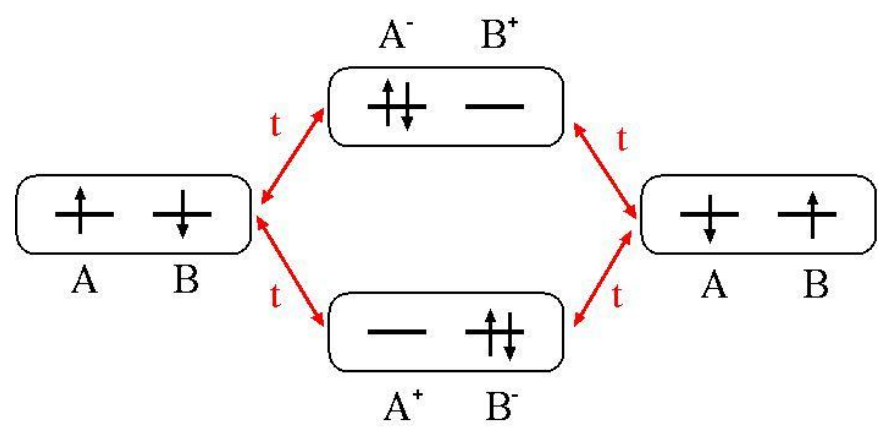

Scheme 1: Neutral and ionic forms involved in the Anderson mechanism. The matrix elements $t$ between the neutral and ionic determinants is indicated in red.

On the contrary, the triplet state cannot be stabilized by the inter-site electron delocalization and its wave-function is only constituted of neutral VB forms. For instance, the Ms=1 spin component wave function is:

$$
{ }^{3} \Psi_{\mathrm{u}}=\mid \text { coregu }|=| \text { core } . \mathrm{ab}
$$

The kinetic exchange always brings an antiferromagnetic contribution to the magnetic coupling and therefore favors the lowest spin state. Working only in the space constituted of neutral and ionic VB forms $|a \bar{b}|,|b \bar{a}|,|a \bar{a}|$ and $|b \bar{b}|$, it is possible to show that the perturbative second-order analytical expression of the magnetic coupling is:

$$
\mathrm{J}=\Delta \mathrm{E}_{\mathrm{ST}}=\mathrm{E}_{\mathrm{S}}-\mathrm{E}_{\mathrm{T}}=2 \mathrm{~K}-4 \frac{\mathrm{t}^{2}}{\mathrm{U}}
$$

When the inter-site delocalization proceeds through the tails of the magnetic MOs on the bridging ligand, this magnetic interaction is called super-exchange and the electronic mechanism is known as the Anderson generalized mechanism which is represented in scheme 2. The electrons jump from one site to the other through the ligand orbitals. 


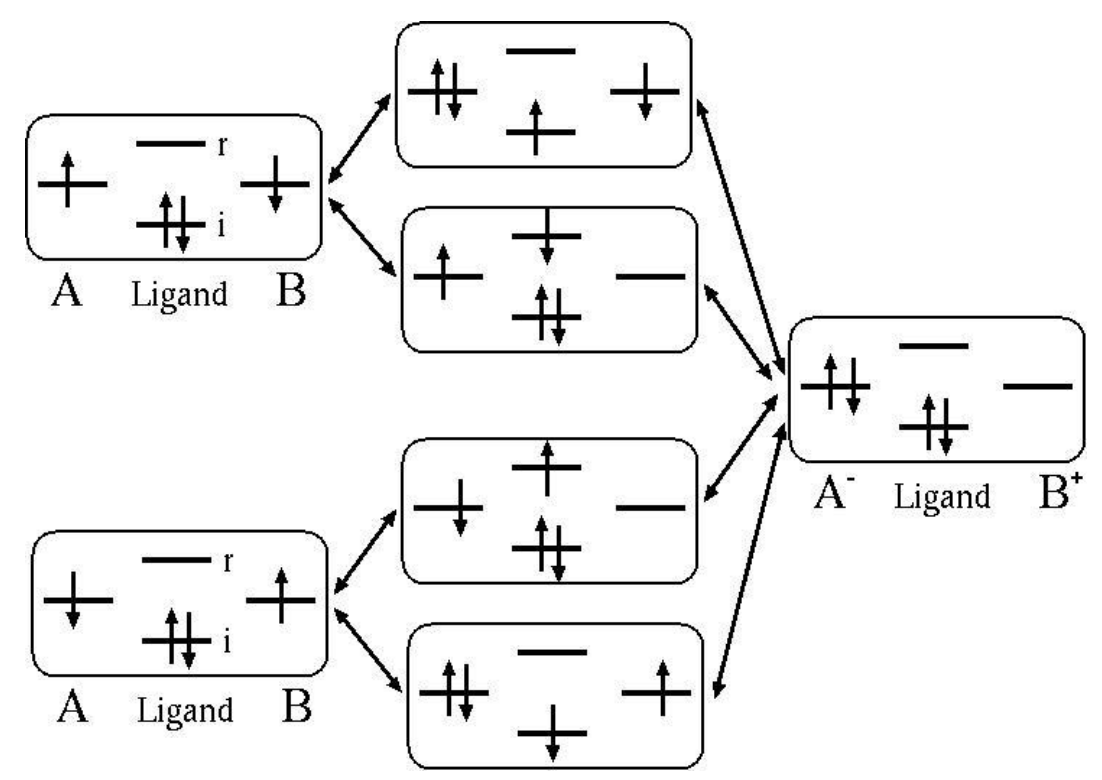

Scheme 2: Configurations involved in the Anderson generalized mechanism. Only one of the two ionic forms is represented. The matrix elements (not indicated) are hopping integrals between the magnetic centers and the ligands.

Different pathways coupling the neutral forms can be proposed, depending on the involved intermediate configurations either ligand to metal charge transfer (LMCT) and/or metal to ligand charge transfer (MLCT). One may note that the Anderson generalized mechanism also involves ionic forms as intermediate steps.

Electron correlation deeply affects the above-mentioned interactions. When using correlated wave function based calculations, several types of excitations contribute to the differential stabilizations of the valence bond determinants (ionic versus neutral) and their interactions. Several papers [9-11] have been devoted to the detailed analysis of these correlation effects. The correlated wave function is expanded on a very large number (millions) of determinants resulting from excitations from the closed shell MOs to the unoccupied ones. As it will be shown below, it is possible to concentrate this information in the sub-space $S_{0}$ defined by the neutral and ionic VB forms, using the theory of effective Hamiltonians [20]. It consists in defining effective interactions between the neutral and ionic VB determinants such that i) the eigenvalues of the effective Hamiltonian matrix are those of the all-electron exact electronic Hamiltonian, i.e. they are equal to the correlated $a b$ initio energies and ii) the eigenvectors of the effective matrix are the projections of the $a b$ initio wave functions onto the sub-space $\mathrm{S}_{0}$. The resulting effective interactions, noted here-after $\mathrm{K}^{\text {eff }}, \mathrm{t}^{\text {eff }}$ and $\mathrm{U}^{\text {eff }}$, take into account the effect of electron correlation. 
Let us distinguish two classes of correlation effects: the first one affects the $\mathrm{K}$ exchange integral while the second one modifies the kinetic contribution to the magnetic coupling.

i) Correlation effect on the exchange integral $\mathrm{K}$ : the spin polarization of the doubly occupied MOs.

The spin polarization mechanism is a real physical phenomenon. It is for instance responsible for the appearance of local spin densities $(\beta)$ which are opposite to the dominant one $(\alpha)$ in $\mathrm{Ms}=1 / 2$ radicals. Its first manifestation, evidenced in the EPR spectroscopy of the $\mathrm{CH}_{3}$ radical, was the non intuitive appearance of a contact term between the spin of the unpaired electron of the carbon and the protons spins. Since the protons are located in the (XY) plane which is a nodal plane for the singly occupied $2 \mathrm{p}_{z}$ orbital bearing the unpaired electron, a zero contact term was expected. Its appearance comes from the spin polarization of the $\alpha$ and $\beta$ spin electrons of the $\sigma$ bonds. Due to a weaker repulsion between electrons having the same spin, the $\alpha$ spins of the $\sigma$ bonds go closer to the carbon while the $\beta$ spins go closer to the protons, generating the contact term between the nuclear spin and the induced electronic $\beta$ spin density.

As shown in previous papers [9], the spin polarization mechanism in diradicals goes through excited configurations involving a triplet state on the bridge and a triplet state in the magnetic orbitals as shown in scheme 3. Since this mechanism never involves any ionic VB configuration, its effect on the magnetic coupling results in a modification of the interaction between the neutral determinants, i.e. a modification of the exchange integral $\mathrm{K}$ such that:

$$
\mathrm{K}^{\mathrm{eff}}=\mathrm{K}+2 \sum_{\mathrm{i}, \mathrm{r}} \frac{\left\langle\mathrm{i}\left|\mathrm{K}_{\mathrm{a}}\right| \mathrm{r}\right\rangle\left\langle\mathrm{r}\left|\mathrm{K}_{\mathrm{b}}\right| \mathrm{i}\right\rangle}{\varepsilon_{\mathrm{r}}-\varepsilon_{\mathrm{i}}}
$$

where $\mathrm{i}$ and $\mathrm{r}$ are respectively occupied and vacant MOs of the ligand and the integral $\left\langle\mathrm{i}\left|\mathrm{K}_{\mathrm{a}}\right| \mathrm{r}\right\rangle$ is the interaction $\left\langle\mathrm{i}(1) \mathrm{a}(2)\left|\mathrm{r}_{12}^{-1}\right| \mathrm{r}(2) \mathrm{a}(1)\right\rangle$. One should notice that while $\mathrm{K}$ is always positive, $\mathrm{K}^{\mathrm{eff}}$ can be either positive or negative.

A detailed analysis of the physical effects on the spin polarization phenomenon of doubly excited determinants which do not involve any ionic forms is out of the scope of the present study. Let us however mention that their contribution to $\mathrm{K}^{\text {eff }}$ is non-negligible and will be taken into account in the following ab inito calculations. 


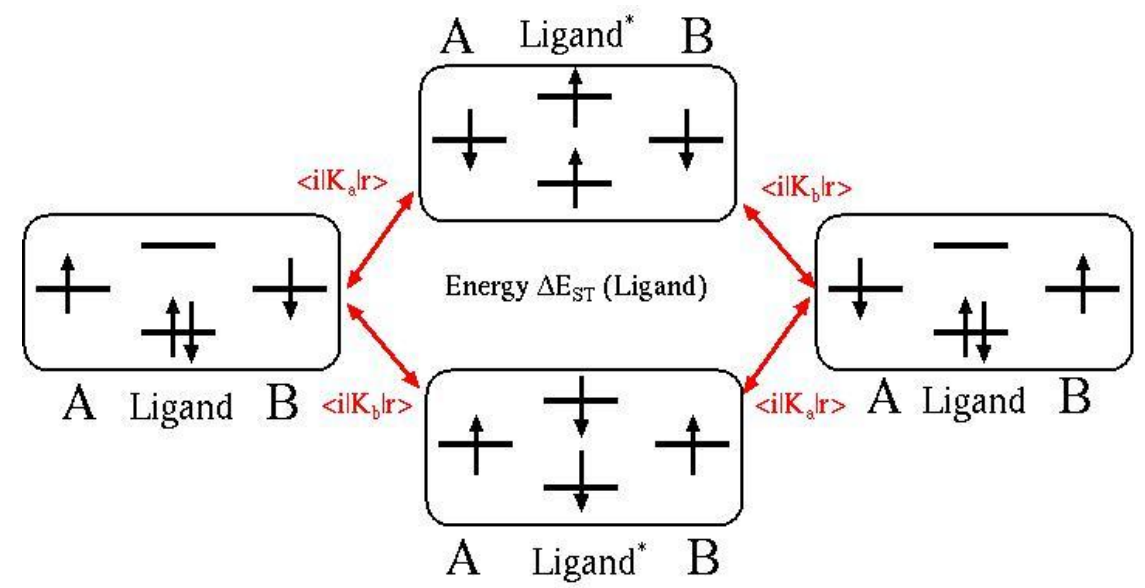

Scheme 3: Configurations involved in the mechanism of the double spin polarization. (Ligand)* means that the ligand is in an excited state, namely a triplet. The matrix elements between the determinants are indicated in red.

ii) Correlation effect on the kinetic exchange : screenings of $U$ and $\triangle E(M L C T / L M C T)$ Single excitations of the non-magnetic electrons are responsible for the so-called charge polarization. This effect reflects the dynamic answer of the non-magnetic electrons to the electric field created in the ionic VB forms (i.e. to the fluctuation of the electric field created by the active electrons in the singlet state). It leads to an effective stabilization of these ionic forms and then to a screening of the on-site repulsion $U$. The correlated value of $U^{\text {eff }}$ can be strongly reduced (by a factor 3 ) in comparison to the uncorrelated one (U) [9-11].

As already shown [9-11], some double excitations also qualitatively affect the kinetic exchange contribution. They are produced by two simultaneous single excitations, either a single excitation on the top of a MLCT (magnetic site to ligand charge transfer) configuration or a single excitation on the top of a LMCT (ligand to magnetic site charge transfer) one. As in the previous case, the single excitation stabilizes the LMCT or MLCT configurations which are important intermediate in the Anderson generalized mechanism. These excitations also increase the delocalization tails between the magnetic and the ligand orbitals [21]. Consequently the hopping integrals $t$ may be changed to $t^{\text {eff }}$ by these double excitations.

II.b Methodological aspects and accurate extraction of the leading effective interactions 
The comparison between the two mechanisms shows that there is no intermediate ionic form in the spin polarization one. As a consequence, it should be possible to distinguish these two contributions by eliminating the ionic forms from the calculation. This subsection describes the required methodology to accurately reproduce magnetic couplings and the procedure implemented in order to separate the two mechanisms.

As shown in equation (4), the zeroth-order singlet wave function is a linear combination of four VB determinants in the localized orbitals $\{a, b\}$ basis. Hence, the zeroth-order description has to be multideterminantal. The singlet and triplet zeroth-order wave functions are computed using the Complete Active Space Self Consistent Field (CASSCF) method, by means of the MOLCAS (version7.2) package[22]. The active space CAS(2,2) treats the interaction of two unpaired electrons in two orbitals while the CASSCF variational procedure optimizes these magnetic orbitals. In order to accurately reproduce magnetic couplings, this description is not precise enough[7-11] and dynamic correlation is introduced using the Difference Dedicated Configuration Interaction (DDCI) method[23] implemented in the CASDI code[24]. DDCI is frequently considered as the best available method for the calculation of magnetic couplings [7-11,25]. It consists in a multireference configuration interaction involving the CAS space and all single and semi-active double excitations on all determinants of the active space. The purely inactive $(2 \mathrm{~h} 2 \mathrm{p})$ excitations are discarded since they do not contribute (to the second order of perturbation) to magnetic couplings.

A modified version of the DDCI method has been implemented in which the coefficients of the determinants corresponding to the ionic forms $\mathrm{A}^{-} \mathrm{B}^{+}$and $\mathrm{A}^{+} \mathrm{B}^{-}$are set to zero along the Davidson diagonalization process. The so-calculated magnetic couplings are the previously defined $\mathrm{K}^{\text {eff }}$ interactions since they only involve spin polarization contributions that modify the direct exchange integral.

In order to extract the effective interactions of the problem, we compute the energy difference between the lowest triplet and singlet states using both the normal DDCI method (J) and the modified version $\left(\mathrm{K}^{\mathrm{eff}}\right)$. Then the intermediate Hamiltonian theory [26] which is a variant of the effective Hamiltonian theory is used in order to extract both $\mathrm{t}^{\mathrm{eff}}$ and $\mathrm{U}^{\mathrm{eff}}$. The intermediate Hamiltonian theory is well-adapted to the considered situation. It consists in using the coefficients $C_{N}$ and $C_{I}$ (see equation (4)) of the singlet ground state onto the model space. The eigenequations obtained for the singlet ground state are: 


$$
\begin{aligned}
& 2 \mathrm{~K}^{\mathrm{eff}} \mathrm{C}_{\mathrm{N}}+2 \mathrm{t}^{\mathrm{eff}} \mathrm{C}_{\mathrm{I}}=\Delta \mathrm{E}_{\mathrm{ST}} \mathrm{C}_{\mathrm{N}} \\
& 2 \mathrm{t}^{\mathrm{eff}} \mathrm{C}_{\mathrm{N}}+\mathrm{UC}_{\mathrm{I}}=\Delta \mathrm{E}_{\mathrm{ST}} \mathrm{C}_{\mathrm{I}}
\end{aligned}
$$

where $\Delta \mathrm{E}_{\mathrm{ST}}=\mathrm{J}$ is computed using the normal DDCI procedure while $2 \mathrm{~K}^{\mathrm{eff}}$ is the energy difference between the singlet and the triplet in the modified DDCI version (without ionic forms). The $\mathrm{t}^{\mathrm{eff}}$ and $\mathrm{U}^{\mathrm{eff}}$ parameters are extracted from the following relations:

$$
\begin{aligned}
& t^{\text {eff }}=\frac{\left(J-2 K^{e f f}\right) C_{N}}{2 C_{I}} \\
& U^{\text {eff }}=J-2 t^{e f f} \frac{C_{N}}{C_{I}}
\end{aligned}
$$

\section{Relative contributions of the Anderson and spin polarization mechanisms to the magnetic coupling}

III.a Definition of a model problem

Calculations have been performed on model compounds where two $\mathrm{CH}_{3}$ radicals are bridged by a neutral polyphenyl chain with external nytril groups. In order to measure the decay of the interactions with the inter-magnetic-site distance, model compounds involving four bridges having either $\mathrm{x}=0,1,2$ and 3 phenyls have been studied (see Scheme 5).

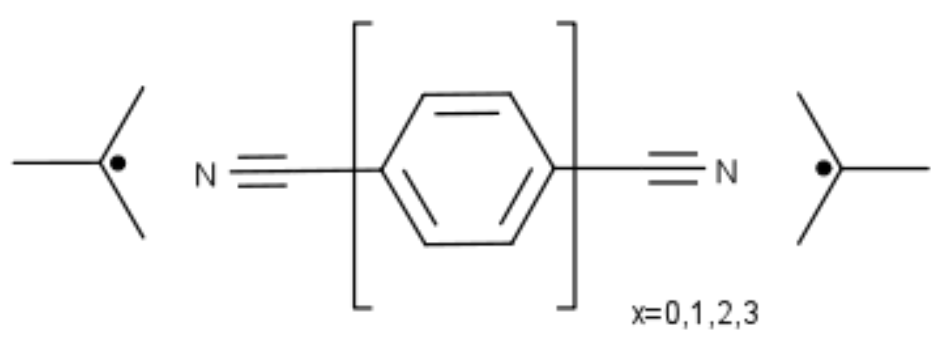

Scheme 5: Schematic representation of the studied model compounds. $x$ refers to the number of phenyls.

The effects of the core electrons $\left(1 \mathrm{~s}^{2}\right)$ of the $\mathrm{C}$ and $\mathrm{N}$ atoms are modelled using a dedicated effective core potential (ECP) and the Double Zeta plus Polarization (DZP) basis sets were used. Double Zeta basis sets are used for the H atoms [26]. 
Geometries of the bridges are optimized using the B3LYP functional and the DFT method implemented in the Gaussian package [28]. In order to keep sizeable values of the magnetic couplings for long bridges, i) the geometries have been constrained to planarity during the optimization process, ii) the distance between the magnetic carbon and the nitrogen is kept at $1.85 \AA$. The magnetic p orbitals are perpendicular to the molecular plane.

\section{III.b Results and discussion}

As expected from the Ovchinnikov's rule [29] all these compounds are antiferromagnetic and the magnetic coupling decreases rapidly with the length of the bridging ligand.

Magnetic couplings calculated for the different model compounds including ( $\mathrm{J})$ and excluding $\left(2 \mathrm{~K}^{\mathrm{eff}}\right)$ the ionic forms are reported in Table I, together with the relative contributions (direct exchange, kinetic exchange and spin polarization) to the final couplings. The direct exchange integral $\mathrm{K}$ (in absence of dynamic correlation) is the exchange integral between the neutral determinants in the CASSCF calculation. While it represents $7 \%$ of $\mathrm{J}$ for $\mathrm{x}=0$, it is only $3 \%$ of $\mathrm{J}$ for $\mathrm{x}=1$ and becomes negligible for longer ligands.

The spin polarization contribution is antiferromagnetic ( $\mathrm{K}^{\mathrm{eff}}$ is negative), in agreement with the Ovchinnikov's rule, since the ligand involves an even number of sites. Its magnitude is larger than that of the direct exchange $\mathrm{K}$ as expected.

The main qualitative result of this study is the varying percentage of the relative contributions of the Anderson and spin polarization mechanisms as function of the bridge length. While magnetic couplings are dominated by the kinetic exchange ( 91\%) for short-length bridges, the relative contribution of spin polarization increases with the length of the bridge and reaches $(\sim 49 \%)$ for $x=3$. One may therefore expect that for longer bridges this contribution is dominant.

\begin{tabular}{|l|l|l|l|l|l|}
\hline$x$ & $J$ & $2 K^{\text {eff }}$ & $2 K$ & Spin polarization & Kinetic exchange \\
\hline 0 & -391.64 & -37.18 & $27.33(-7 \%)$ & $-64.51(16.5 \%)$ & $-4 \mathrm{t}^{\mathrm{eff}}{ }^{2} / \mathrm{U}^{\mathrm{eff}}$ \\
\hline 1 & -52.64 & -13.62 & $1.60(-3 \%)$ & $-15.22(28.9 \%)$ & $-39.02(74.1 \%)$ \\
\hline
\end{tabular}




\begin{tabular}{|l|l|l|l|l|l|}
\hline 2 & -7.30 & -2.83 & $0.14(-1.9 \%)$ & $-2.97(40.7 \%)$ & $-4.47(61.2 \%)$ \\
\hline 3 & -1.04 & -0.49 & $0.02(-1.9 \%)$ & $-0.51(49 \%)$ & $-0.55(52.9 \%)$ \\
\hline
\end{tabular}

Table I: Magnetic couplings $\mathrm{J}$ and $2 \mathrm{~K}^{\text {eff }}$ (in $\mathrm{cm}^{-1}$ ) computed using the DDCI method with and without ionic determinants respectively and the different contributions (in $\mathrm{cm}^{-1}$ ) to the magnetic coupling, namely the direct exchange $\mathrm{K}$, the spin polarization and the kinetic exchange contributions. Percentages of $\mathrm{J}$ brought by the different contributions are also indicated.

Parameters $\mathrm{t}^{\mathrm{eff}}$ and $\mathrm{U}^{\mathrm{eff}}$ extracted using equation (9) are reported in Table II. These results call the following comments:

i) the effective hopping integral decreases exponentially with the distance

ii) $U^{\text {eff }}$ increases, as expected from the law $C-1 / R$ where $C$ is a constant and $R$ is the distance between the plus and minus charges in the ionic function.

The variation of the quantities $\ln \left(\left|\mathrm{t}^{\mathrm{eff}}\right|\right), \quad \ln \left(\mathrm{K}^{\mathrm{eff}}\right), \quad \ln (|\mathrm{J}|), \quad \ln \left(4\left(\mathrm{t}^{\mathrm{eff}}\right)^{2} / \mathrm{U}\right)$ and $\ln \left(\left|\mathrm{E}_{\mathrm{SP}}\right|\right)$ (where $\mathrm{E}_{\mathrm{SP}}=2 \mathrm{~K}^{\text {eff }}-2 \mathrm{~K}$ is the spin polarization contribution) are represented as functions of the inter-magnetic-site distances in Figure 1. Expected exponential decays are observed. The slopes are $\gamma=-0.241$ and $\gamma^{\prime}=-0.455$ for $\ln \left(\left|\mathrm{t}^{\mathrm{eff}}\right|\right)$ and $\ln (|\mathrm{J}|)$ respectively, resulting in a ratio of $\gamma^{\prime} / \gamma=1.89$, slightly lower than 2 . The deviation to the ratio 2 expected from the Anderson mechanism, should of course be attributed to the spin polarization contribution. The slope of $\ln \left(\left|E_{S P}\right|\right), \gamma "=-0.372$, is actually smaller in absolute value than the one of $2 \ln \left(\left|t^{\text {eff }}\right|\right)$, which is consistent with the dominant contribution of the spin polarization mechanism for longdistance couplings. Finally, one sees that when the length of the conjugated bridges increases, the $\mathrm{J}$ curve moves away from the $4\left(\mathrm{t}^{\mathrm{eff}}\right)^{2} / \mathrm{U}$ one and goes closer to the SP curve which reflects the asymptotic prevalence of spin polarization in the magnetic coupling.

\begin{tabular}{|c|c|c|}
\hline $\mathbf{X}$ & $\mathbf{t}^{\text {eff }}\left(\mathrm{cm}^{-1}\right)$ & $\mathbf{U}^{\text {eff }}\left(\mathrm{cm}^{-1}\right)$ \\
\hline 0 & -2407 & 61534 \\
\hline 1 & -835 & 71528 \\
\hline 2 & -292 & 76301 \\
\hline
\end{tabular}




\begin{tabular}{|l|l|l|}
\hline 3 & -104 & 78855 \\
\hline
\end{tabular}

Table II: $\mathrm{t}^{\mathrm{eff}}$ and $\mathrm{U}^{\mathrm{eff}}$ parameters extracted from the intermediate Hamiltonian theory for compounds with $\mathrm{x}=0,1,2$ and 3 .

\section{Conclusion and perspectives}

$A b$ initio calculations have been performed in order to calculate the magnetic couplings of a consistent series of bi-centered magnetic model compounds. At the first glance, the behavior of these interactions as function of the inter-magnetic-site distance would be governed by the Anderson generalized mechanism. Nevertheless a deeper inspection reveals that the spin polarization mechanism provides a non-negligible contribution.

Owing to a modification of the CI method, namely the removal of ionic configurations from the CI space, it has been possible to eliminate the kinetic exchange contribution to the magnetic coupling and therefore the Anderson mechanism. The intermediate Hamiltonian theory enables one to quantify all the interactions governing the magnetic exchange, i.e. the hopping integral, the relative energy of ionic configurations, the direct exchange and the spin polarization contribution to the magnetic coupling. From these interactions it becomes possible to quantify both the spin polarization and kinetic exchange contributions. The hopping integral, magnetic coupling and spin polarization contribution exhibit an exponential decay with the inter-magnetic-site distance. The straight line of the $\ln \left(\left|\mathrm{t}^{\mathrm{eff}}\right|\right), \ln (\mathrm{J})$ and $\ln \left(\mathrm{K}^{\mathrm{eff}}\right)$ show different slopes. Due to the spin polarization contribution to the magnetic couplings, the ratio (1.89) between the slopes of $\ln \left(\left|\mathrm{t}^{\mathrm{eff}}\right|\right)$ and $\ln (\mathrm{J})$ is different from the value 2 expected from the Anderson mechanism. Finally, it is shown that the spin polarization mechanism becomes the leading contribution for long-distance magnetic couplings. This conclusion supports the proposition of ref[14,15], which was based on the appearance of spin densities in brokensymmetry UDFT calculations. A methodological effort allowing one to separate the spin polarization from the kinetic exchange in UDFT calculations is conceivable and would be welcome. For the time being the wave function based methods present the advantage of giving access to a decomposition of the mechanisms contributing to the studied property, with a possible identification of interferences between them.

Another important conclusion of this work is the slower decrease of spin polarization with the inter-magnetic site distance in comparison to the kinetic exchange contribution. One may 
therefore expect that long ligands highly spin polarizable, such as polyacene[30] would induce a good magneto-communication between magnetic units.

As reference [16], the present work questions the prevalence of the Anderson mechanism in the magnetic coupling. Nevertheless, while the present study rationalizes the appearance of a ratio slightly different from 2 between the slopes of $\ln \left(\left|t^{\text {eff }}\right|\right)$ and $\ln (J)$, it does not provide any explanation for the obtained ratio $\sim 0.5$ from experimental measurements of reference [16], since the overall ratio slope of the magnetic coupling is 1.89 . A remaining question concerns the possible difference between the values of $t$ and $\mathrm{V}_{\mathrm{AB}}$. Moreover, the experimental determination of the hopping integral in mixed valence systems involving long bridging ligands, i.e. where the extra electron is localized on one metal ion, is far from being direct. Its extraction requires several approximations (known as the Hush's model[17]) and the environment (polar solvent) effects may qualitatively affect the $\mathrm{V}_{\mathrm{AB}}$ value.

A further work will concentrate on this question, i.e. mixed valence compounds will be studied. Both the effect of the environment (polar solvent) and the validity of the extraction of $\mathrm{V}_{\mathrm{AB}}$ from the spectroscopy of complexes of class III using the Hush's model [17] will be analyzed. 


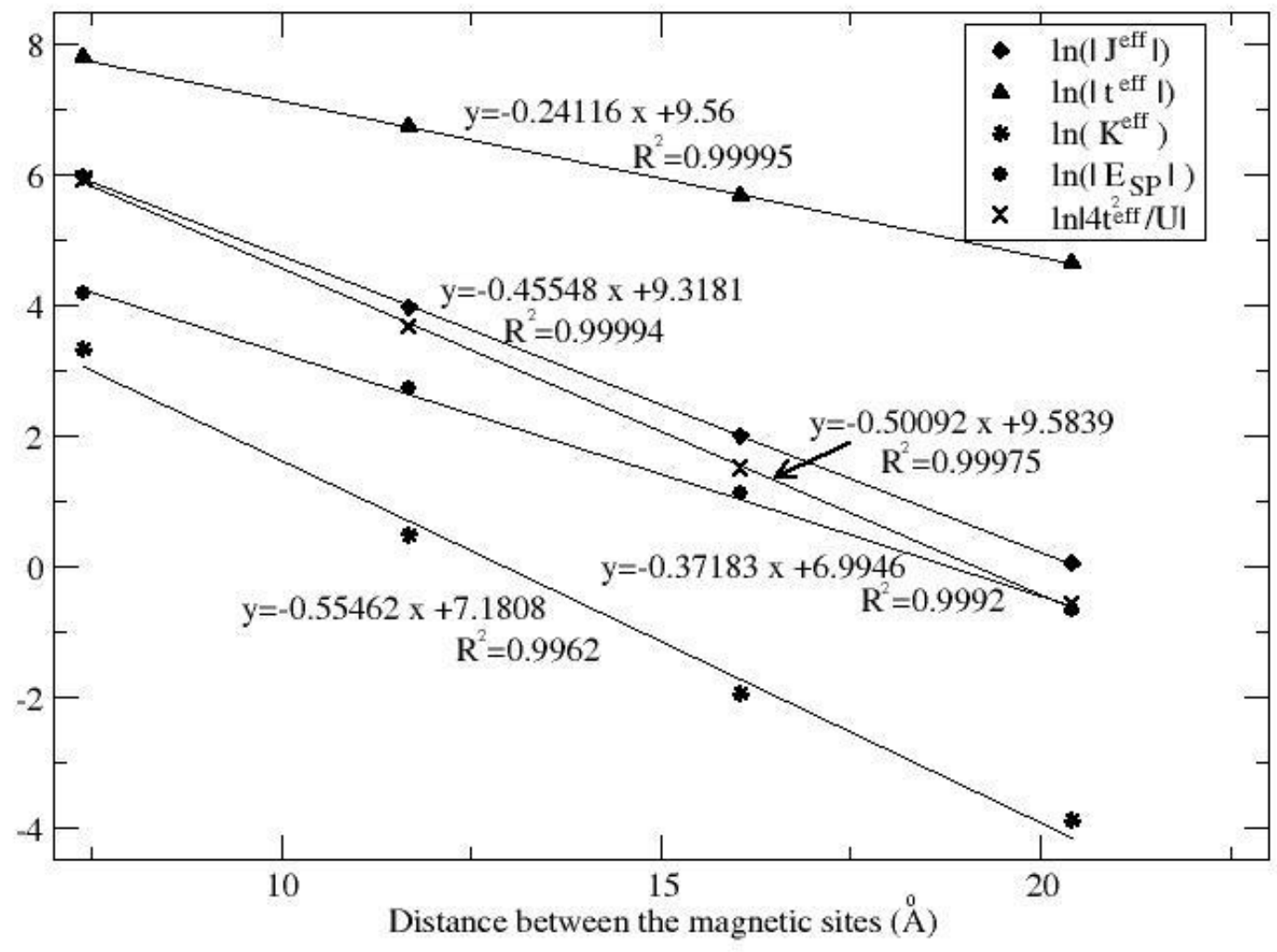

Figure 1: $\ln \left(\left|\mathrm{t}^{\mathrm{eff}}\right|\right), \ln (|\mathrm{J}|), \ln \left(\left|\mathrm{K}^{\mathrm{eff}}\right|\right), \ln \left(4\left(\mathrm{t}^{\mathrm{eff}}\right)^{2} / \mathrm{U}\right)$ and $\ln \left(\left|\mathrm{E}_{\mathrm{SP}}\right|\right)$ as functions of the intermagnetic-site distance. Linear regressions have been performed; the straight-line equations and the correlation coefficients are indicated. 


\section{Bibliography}

(1) Jorner J., Ratner M., (Eds.) Molecular Electronics, Blackwell Science, Oxford (1997)

(2) (a) Joachim C.J., Gimzevski, J. K., Aviram A., Nature 408, 541 (2000)

(b) Dei A., Gatteschi D., Sangregorio C., Sorace L., Acc. Chem. Res. 37, 827 (2004)

(c) Hamon P., Justaud F., Cador O., Hapiot P., Rigaud S., Toupet 1., Ouahab L., Stueger H., Hamon J-R., Lapinte C., J. Amer. Chem. Soc. 130, 17372 (2008)

(d) Lafferentz L., Ample F., Yu H., Hecht S., Joachim C., Grill L., Science 323, 1193 (2009)

(3) Launay J.-P. Chem. Soc. Rev. 30, 386 (2001)

(4) Launay J.-P., Coudret C. Electron Transfer in Chemistry; Wiley-VCH 5, 3 (2001)

(5) Gatteschi D., Sessoli R.,Villain J., Molecular Nanomagnets Oxford University Press, New York (2006)

(6) Ardavan A., Rival O., Morton J.L.L., Blundell S.J., Tyryshkin A.M., Timco G. A., Winpenny R.E.P., Phys. Rev. Lett. 98, 057201 (2007)

(7) Bencini A, Inorg. Chim. Acta 361, 3820 (2008)

(8) Moreira I. de P.R. and Illas F., Phys. Chem. Chem. Phys. 8, 1645 (2006)

(9) Calzado C.J., Cabrero J., Malrieu J-P., Caballol R., J. Chem. Phys. 116, 2728 (2002)

(10) Calzado C.J., Cabrero J., Malrieu J-P., Caballol R., J. Chem. Phys. 116, 3985 (2002)

(11) Calzado C.J., Angeli C., Taratiel D., Caballol R., Malrieu J-P., J. Chem. Phys. 131, 044327 (2009)

(12) Ali M. E., Datta S. N., J. Phys. Chem. A 110, 2776 (2006), Ali M. E., Datta S. N., J. Phys. Chem. A 110, 13232 (2006)

(13) Lahti P.M., Ichimura A.S., Sanborn J.A., J. Phys. Chem. A 105, 251 (2001)

(14) Pardo E., Carrasco R. Ruiz-Garcia R., Julve M., LLoret F., Munoz MC, Journaux Y., Ruiz E., Cano J. J. Am. Chem. Soc. 130, 576 (2008)

(15) Ferrando-Soria J., Castellano M., Yuste C., Lloret F., Julve M., Fabelo O., Ruiz-Pérez C., Stiriba S-E., Ruiz-García R., Cano J., Inorg. Chim. Acta 363, 1666 (2010)

(16) Fabre M., Bonvoisin J., J. Am. Chem. Soc. 129, 1434 (2007)

(17) Hush N. S., Coord. Chem. Rev. 64, 135 (1985)

(18) Kahn, O. Molecular Magnetism; Wiley-VCH (1993)

(19) (a) Anderson, P. W. Phys. Rev. 79 (2), 350 (1950)

(b) Anderson, P. W. Phys. Rev. 115, 2 (1959) 
(c) Anderson, P. W. In Magnetism, Rado, G. T., Suhl, H., Eds. Academic Press: New York 1, $25(1965)$

(20) for a review see Durand P. Malrieu J-P., in Ab initio Quantum Chemistry, Lawley Ed. (Wiley and sons) 1, 321 (1986)

(21) Cabrero J., Calzado C. J. Maynau D. Caballol R., Malrieu J-P., J. Phys. Chem. A 106 $8146(2002)$

(22) Molcas package, version 7, G. Karlström, R. Lindh, P.- $\mathrm{A}$. Malmqvist, B. O. Roos, U. Ryde, V. Veryazov, P.-O. Widmark, M. Cossi, B. Schimmelpfennig, P. Neogrady, L. Seijo, Computational Material Science, 28, 222 (2003).

(23) (a) Miralles J., Castell O., Caballol R., and Malrieu J. P., Chem. Phys. 172, 33 (1993)

(b) Miralles J., Daudey J. P., and Caballol R., Chem. Phys. Lett. 198, 555 (1992)

(24) CASDI program, Ben Amor N. and Maynau D., Chem. Phys. Lett. 286, 211 (1998)

The method is also implemented in the SORCI program of the ORCA code, Neese F., J. Chem. Phys. 119, 9428 (2003)

(25) Barone V., Cacelli I., Ferretti A., Prampolini G., J. Chem. Phys. 13, 224103 (2009)

(26) Malrieu J. P., Durand P., Daudey J. P., J. Phys. A, Math. Gen. 18, 809 (1985)

(27) (a) Bergner A., Dolg M., Kuechle W., Stoll H., Preuss H., Mol. Phys. 80, 1431 (1993);

(b) Widmark P.-O., Malmqvist P.-A., and Roos B. O., Theor. Chim. Acta 77, 291, (1990).

(28) Gaussian 09, Revision A.1, M. J. Frisch, G. W. Trucks, H. B. Schlegel, G. E. Scuseria, M. A. Robb, J. R. Cheeseman, G. Scalmani, V. Barone, B. Mennucci, G. A. Petersson, H. Nakatsuji, M. Caricato, X. Li, H. P. Hratchian, A. F. Izmaylov, J. Bloino, G. Zheng, J. L. Sonnenberg, M. Hada, M. Ehara, K. Toyota, R. Fukuda, J. Hasegawa, M. Ishida, T. Nakajima, Y. Honda, O. Kitao, H. Nakai, T. Vreven, J. A. Montgomery, Jr., J. E. Peralta, F. Ogliaro, M. Bearpark, J. J. Heyd, E. Brothers, K. N. Kudin, V. N. Staroverov, R. Kobayashi, J. Normand, K. Raghavachari, A. Rendell, J. C. Burant, S. S. Iyengar, J. Tomasi, M. Cossi, N. Rega, J. M. Millam, M. Klene, J. E. Knox, J. B. Cross, V. Bakken, C. Adamo, J. Jaramillo, R. Gomperts, R. E. Stratmann, O. Yazyev, A. J. Austin, R. Cammi, C. Pomelli, J. W. Ochterski, R. L. Martin, K. Morokuma, V. G. Zakrzewski, G. A. Voth, P. Salvador, J. J. Dannenberg, S. Dapprich, A. D. Daniels, O. Farkas, J. B. Foresman, J. V. Ortiz, J. Cioslowski, and D. J. Fox, Gaussian, Inc., Wallingford CT, 2009.

(29) Ovchinnikov A.A., Theoret. Chim. Acta 47, 297 (1978)

(30) Hachmann J., Dorando J.J., Aviles M., Chan G.K.L, J. Chem. Phys. 127, 134309 (2007) 